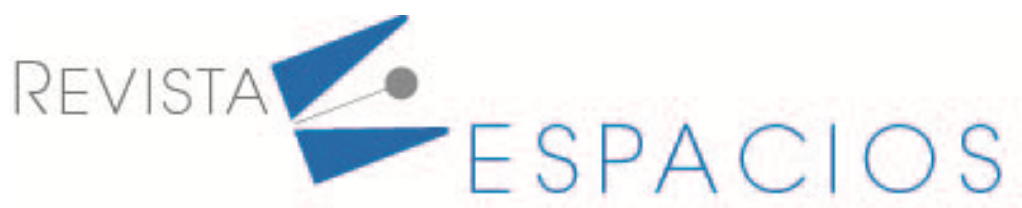

\title{
Efecto de la aglomeración en la productividad de los sectores industriales en el departamento de Antioquia- Colombia
}

\section{Effect of the agglomeration on the productivity of industrial sectors in the department of Antioquia-Colombia}

\author{
ALARCÓN, Victoria ${ }^{1}$ \\ GUTIERREZ, Carlos E. ${ }^{2}$ \\ FUENTES, HECTOR J. ${ }^{3}$
}

\begin{abstract}
Resumen
El presente trabajo analiza la relación entre aglomeración y productividad para el departamento de Antioquia en el ańo 2017. Se evalúan las interacciones por actividad industrial para validar la presencia de aglomeraciones a través de un análisis de estadística espacial de patrones puntuales. Se obtienen las actividades aglomeradas, que representan la mitad de las actividades industriales, y se construye un modelo a partir de una función tipo Cobb Douglas para las actividades con patrón de aglomeración. La importancia de este procedimiento metodológico radica en la posibilidad de calcular las productividades asociadas al trabajo y al capital de las industrias y determinar el tipo de rendimientos de escala presentes en estas.
\end{abstract}

Palabras clave: aglomeración, función Cobb Douglas, patrones puntuales, productividad

\begin{abstract}
This paper analyzes the relationship between agglomeration and productivity for the department of Antioquia in 2017. Interactions by industrial activity are evaluated to validate the presence of agglomerations through a spatial statistics analysis of specific patterns. The agglomerated activities are obtained, which represent half of the industrial activities, and a model is constructed from a Cobb Douglas type function for activities with agglomeration pattern. The importance of this methodological procedure lies in the possibility of calculating the productivities associated with labor and the capital of the industries and determining the type of scale returns present in them

key words: agglomeration, productivity, Cobb Douglas function, point patterns
\end{abstract}

\section{Introducción}

La localización industrial además de ser estudiada desde el punto de vista hermenéutico del espacio, permite que sus implicaciones puedan medirse también desde lo económico. Partiendo del supuesto de que la aglomeración industrial pueda tener algún efecto en el desempeño económico, surge la idea de evaluar si la

\footnotetext{
${ }^{1}$ Estudiante Ingenieria catastral y geodesia. Universidad Distrital Francisco José de Caldas, Bogotá, mvalarconh@correo.udistrital.edu.co

${ }^{2}$ Economista. Estudiante Ingeniería catastral y geodesia. Universidad Distrital Francisco José de Caldas, Bogotá, cegutierrezu@correo.udistrital.edu.co

${ }_{3}^{3}$ Profesor titular Facultad de ingeniería. Universidad Distrital Francisco José de Caldas, Bogotá, hjfuentesl@udistrital.edu.co
} 
configuración de clústeres industriales bajo unas dinámicas propias de cada actividad, genera unos beneficios económicos medidos por medio de su productividad en términos de capital y trabajo. La metodología empleada permite evaluar sobre la industria en Antioquia para el año 2017, la existencia de aglomeración tipo clúster en los diferentes sectores industriales. Posteriormente, aquellas actividades que cumplieron con la existencia de aglomeración pasaron a la siguiente etapa que consiste en la medición de su productividad a través de la función de producción de Cobb Douglas. Los resultados obtenidos sugieren que de las 605 empresas que ejercen actividades industriales, alrededor de la mitad desarrollan sus actividades aglomeradas, intensivas en mano de obra, con rendimientos decrecientes a escala en unos casos y en otros, muy cerca de generar rendimientos constantes a escala. La evidencia nos muestra que la industria se localiza con mayor profundidad en el área metropolitana del valle de Aburrá, siendo más predominante en la ciudad de Medellín.

\subsection{Antececentes}

El análisis de las aglomeraciones industriales es un tema de continua relevancia y de importante interés por parte de los investigadores. Varios de los énfasis en torno a la literatura existente buscan explicar como estas aglomeraciones se han ido formado en torno a atractores de diversa índole que incentivan a las empresas a ubicarse en un determinado lugar. Al respecto en términos de la literatura internacional se destacan los trabajos de (Holl, 2004), (Lee, 2006) y (Lee, 2008), (Sleuwaegen \& Pennings, 2006), (Pellenbarg et al., 2002), (Brouwer et al., 2004), (Artís et al., 2007), (Knoben \& Oerlemans, 2008) y (Lampón, 2010), para explicar la localización y relocalización de las industrias en Europa, Estados Unidos, y países asiáticos. Estos trabajos identifican patrones que explican movimientos de las industrias al interior de los países, así como movimientos hacia nuevas aglomeraciones a través de los países y del mismo modo como se van generando tanto atractores como expulsores de las industrias que cambian el espacio físico de las empresas buscando lugares más eficientes en términos competitivos y acceso a nuevos mercados.

A nivel local se encuentran trabajos como los de (Ospina Vazquez, 1987), (Ocampo, 1984), o (Safford, 1965, 1989) son ya referentes clásicos del proceso de industrialización en el país. Un poco más recientes se destacan los trabajos de (Londoño, 2015), (Toro González, 2004), así como los de (Camilo Murcia et al., 2014; Fuentes et al., 2019) explican los procesos de localización al interior del país y sus movimientos y transformaciones espaciales que se convienen en referentes nacionales de la relación territorio e industria. Se destacan de estos trabajos como se identifican los procesos de localización, relocalización, así como de la formación de industrias y su relación con los procesos regionales que identifican las fuerzas expulsoras y atractoras que han ido formando y transformando las aglomeraciones existentes en términos industriales. Estos referentes tanto externos como internos han servido como punto de apoyo tanto desde el punto metodológico como teórico para el análisis propuesto en este trabajo.

\subsection{Marco Teórico}

Todas las referencias teóricas de la localización de las actividades económicas parten de la explicación dada por (Von Thünen, 1826) quien experimentó diferentes métodos de localización racional de sus cultivos. Como lo expresa (Wood \& Roberts, 2011) Thünen consideró quizá la más básica de las preguntas hechas por los geógrafos económicos: ¿por qué cierta actividad toma lugar en cierto lugar particular? El modelo de Von Thünen desarrolló dos hilos principales. El primero concentrado en mejorar la productividad a través de un entendimiento de la economía de los cultivos en términos de intensidad y variedad y el segundo enfocado en el cálculo de los salarios. En este segundo punto el tema de los salarios naturales fue concebido como un vínculo en productividad y pago. No obstante, para la geografía siempre fue más interesante su esquema acerca del uso de la tierra agrícola. En este esquema se parte de un plano isotrópico con un único centro poblado alrededor del cual se localizan de manera circular diferentes productos agrícolas, con diferentes características. Por ejemplo, qué tan perecedero y qué tan fácil de transportar es el producto. El modelo propuesto por Thünen buscaba explicar la renta de la 
tierra en función del precio de los productos, menos los costos de producción y el transporte al mercado. Para el modelo de Thünen la agricultura intensiva estaría cerca del centro o del mercado y una agricultura extensiva con más altos costos de transporte estaría ubicada más lejos del centro urbano (Wood \& Roberts, 2011).

\section{Gráfico 1}

Uso de la tierra rural y urbana basado en el modelo de Thünen
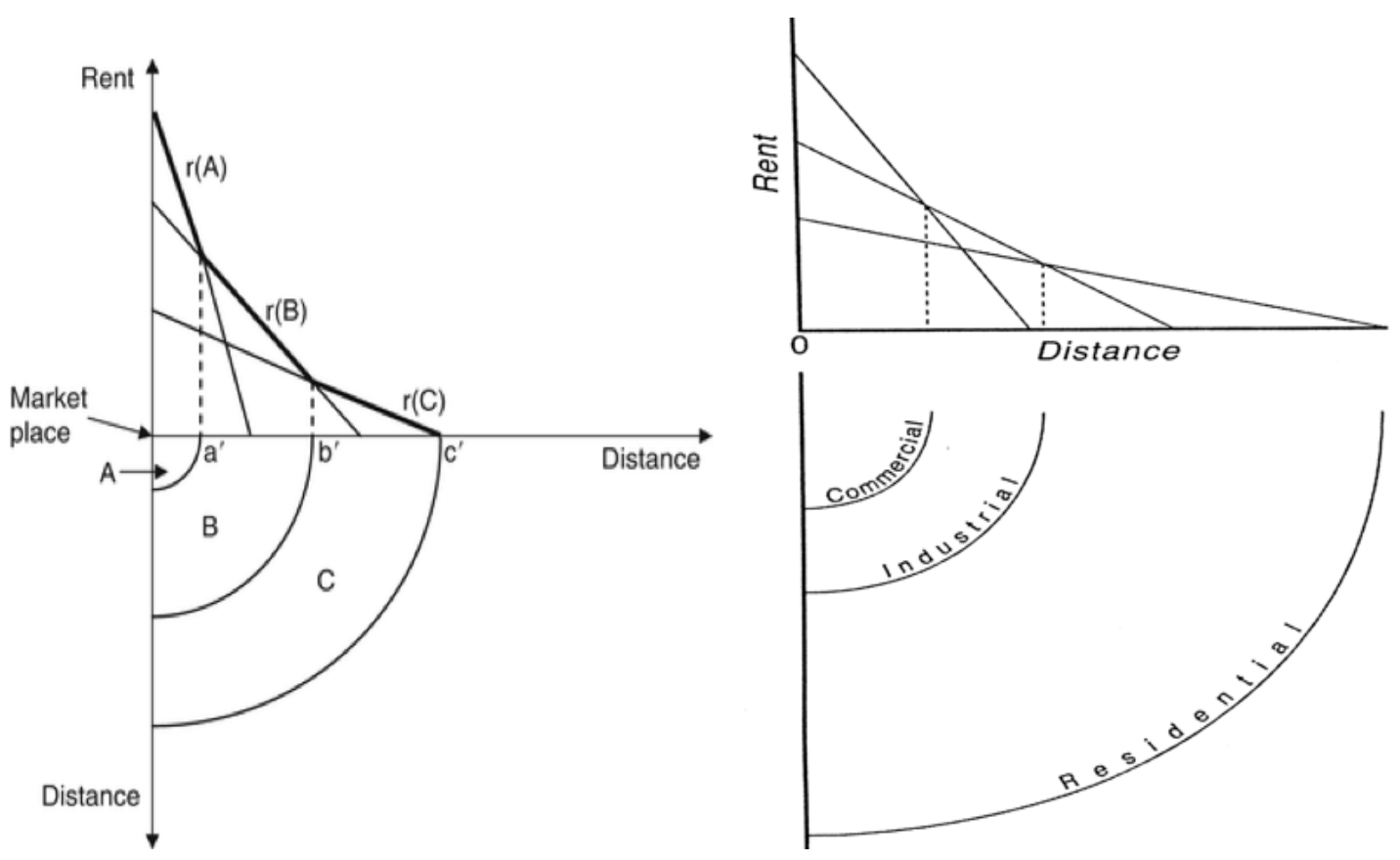

Fuente: (Capello, 2014) y (Wood \& Roberts, 2011)

El gráfico 1 muestra la representación del modelo de Thünen en donde la relación renta y distancia definen el tipo de cultivo que se siembra alrededor del centro urbano o de mercado. Este mismo análisis ha sido adaptado para referirse a la conformación de círculos concéntricos basados en la distancia en la conformación de ciudades (Fuentes et al., 2019). Aquí en lugar de diferentes tipos de cultivos se representan sectores económicos como comercio, industria y vivienda.

Una segunda referencia a la localización industrial la realiza (Weber, 1929), quien postula su modelo de costo mínimo de localización industrial. Este modelo se asume que la localización más racional depende de los costos más bajos. Para Weber los costos de transporte, tanto de insumos como de bienes finales, son una función del peso y de la distancia desde las fuentes donde se obtienen (materias primas) y hacia el mercado donde se venden (bienes terminados). 
Gráfico 2

Modelo de localización industrial de WEBER

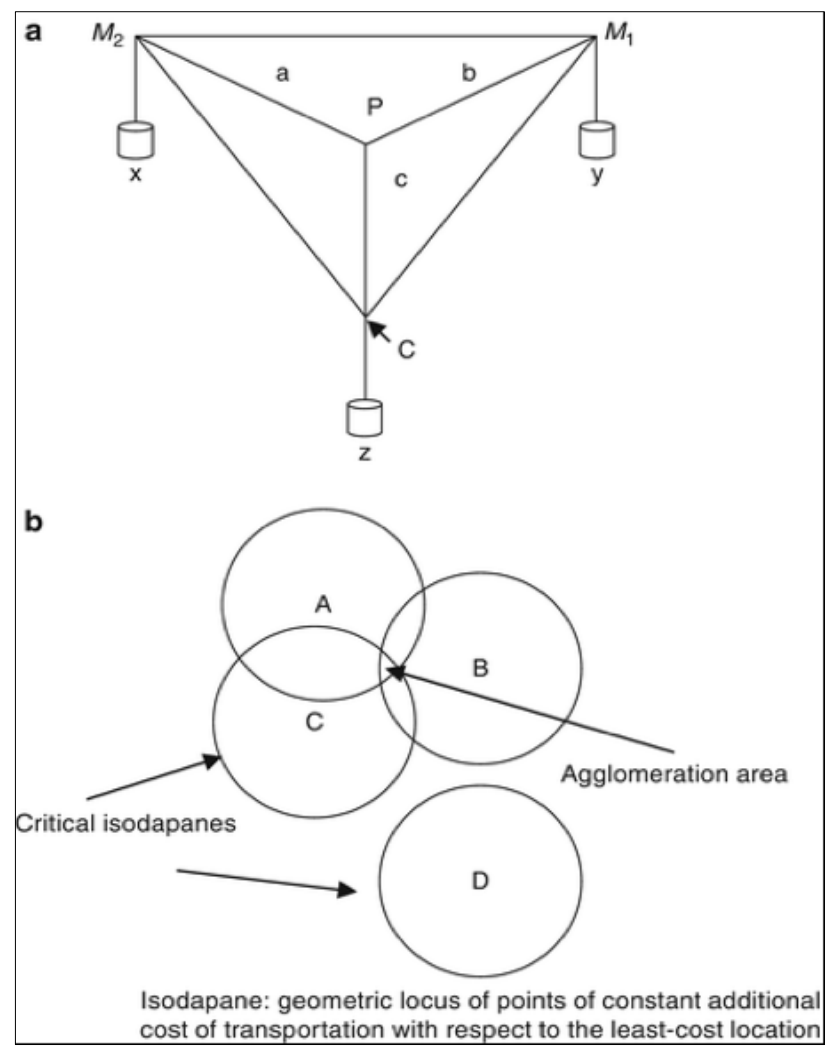

Fuente: (Capello, 2014)

En el gráfico 2a se representa en el triángulo de Weber donde a, b y c representan las distancias en kilómetros entre los mercados de materias primas y el sitio de producción, así como entre este último y el mercado final. Por su parte $x a, y b, y z c$ representan las fuerzas de atracción que empujan la empresa respectivamente hacia los puntos M1, M2 y C (Capello, 2014).

El gráfico $2 \mathrm{~b}$ representa las isodapas (curvas alrededor del cual los costos de transporte que la firma debe pagar con el propósito de cubrir cierta distancia desde el punto de costo más bajo permanecen constantes) de cada industria y los movimientos que las aglomeran. Las industrias A, B y C encuentran ventajas al relocalizarse y concentrarse pues el mayor costo de transporte es compensado por la ventaja que genera la aglomeración (Capello, 2014). Este no es el caso de la industria D pues la ventaja de aglomerarse no es más alta que el adicional costo de transporte

Luego de considerar los efectos de los insumos, el mercado y los costos de transporte, (Weber, 1929) introdujo los factores laborales. Específicamente, su preocupación giró en torno a las condiciones sobre las cuales en los costos laborales actuarían como un atractor de localización sobre una industria. El modelo de Weber indica que estos movimientos ocurrirían si los ahorros en costos laborales por unidad de producto son mayores que el incremento en el costo del transporte incurridos desde el punto de localización óptimo (Wood \& Roberts, 2011).

\subsection{Industría en Antioquia}

Existe una importante literatura en torno a la industria en Antioquia, que constituye uno de los departamentos con mayor industria después de Bogotá y su Sabana. Antioquia no solo es un departamento con una industria constituida, sino que además fue uno de los pioneros de la industrialización en el país. En (Safford, 1989) y en (Fuentes et al., 2019) se puede evidenciar como en Antioquia se dieron los primeros resquicios de industria pues 
su clase mercantil, generó un proceso institucional que permitió que las élites antioqueñas vieran en la industria, especialmente en la siderurgia y en la producción de otras manufacturas como la industria textil una oportunidad de desarrollo. Al igual que Bogotá, Medellín se constituyó en el centro de la industrialización antioqueña, ayudado por un importante crecimiento poblacional y por los ingresos generados de la economía cafetera que, de alguna manera, sirvieron para cambiar la vocación agrícola por una más enfocada hacia la industria. No obstante, como complemento Botero (1983) citando a Brew (1977), considera que, si bien el financiador del proceso industrializador antioqueño corresponde al Café como eje de esta transformación productiva junto al importante mercado interno, la minería y el comercio jugaron un papel representativo en esta transición, dado que moldearon al hombre de negocios y empresario que se convertiría en industrial. El departamento antioqueño se destaca porque tanto en su ciudad capital Medellín como en su área metropolitana que incluye a municipios como Bello, Itagüi, Sabaneta, Envigado, y Guarne entre otros articuló un complejo industrial de la región en la que se evidencia la existencia de clústers de establecimientos industriales sin desconocer que hacia otras zonas más periféricas del departamento como Caucacia, Apartadó y Carepa también se han dado aglomeraciones alejadas de la ciudad central. Dichas aglomeraciones son las que buscan encontrarse y representarse en el presente artículo.

\section{Metodología}

Los datos empleados en esta investigación provienen del sistema de información y reporte empresarial - SIREM - (Supersociedades, 2018) el cual presenta los estados financieros y gastos de intereses con corte a 31 de diciembre a nivel empresarial del año 2017, suministrados por las empresas que se encuentran sometidas a inspección y vigilancia por la Superintendencia de Sociedades y que pertenecen al sector real de la economía. La base de datos contiene la información general de la empresa como su nomenclatura predial, datos de contacto, fecha de matrícula, Nit, etc. Se destaca que la información contenida en la base de datos desde el año 2016 en adelante se encuentra ajustada a las Normas Internacionales de Información Financiera - NIIF-, donde ya no se emplean los Estados de Resultados y el Balance General, propio de la contabilidad nacional, sino que la norma internacional exige el Estado de Situación Financiera, Estado de Resultados Integrales, entre otros. De estos dos estados contables se sustraen las variables del modelo.

No obstante, los datos fueron filtrados únicamente para el departamento de Antioquia, exclusivamente para actividades industriales. Esta selección de actividades industriales se obtuvo por medio de la clasificación CIIU (Clasificación Industrial Internacional Uniforme), que permite registrar a las empresas de acuerdo a su actividad económica. En total, se registraron 23 actividades industriales, relacionadas con la fabricación, elaboración, confección, coquización de productos, entre otros.

Posteriormente, se realizó la espacialización de las empresas industriales. Este proceso se realizó de manera manual a partir de las nomenclaturas prediales registradas en la base de datos. Las coordenadas obtenidas se georreferenciaron minuciosamente por medio de Google Earth, para determinar la ubicación de la fábrica o planta de producción y no de la oficina administrativa. Como resultado final, 23 actividades registran las más de 605 empresas que ejercen actividades fabriles en el departamento.

Con el fin de obtener una base de datos que en el proceso metodológico no genere problemas con los datos atípicos, datos incompletos, etc.; se procede con la normalización, arrojando finalmente un total de 431 empresas distribuidas en 20 actividades industriales resumidas en la siguiente tabla. 
Tabla 2

Número de empresas según actividad económica en Antioquía

\begin{tabular}{|c|c|c|c|}
\hline $\mathrm{N}^{\circ}$ & Actividad industrial & Clasificación DIAN & Número de empresas \\
\hline 1 & Actividades de impresión & C1811 & 18 \\
\hline 2 & Confección de prendas de vestir & C1410 & 68 \\
\hline 3 & Coquización y fabricación de productos & C1921 & 4 \\
\hline 4 & Curtido cuero y fabricación & C1511 & 10 \\
\hline 5 & Elaboración de productos alimenticios & C1011 & 52 \\
\hline 6 & Fabricación de aparatos y equipo eléctrico & C2740 & 9 \\
\hline 7 & Fabricación de maquinaria y equipo & C2829 & 6 \\
\hline 8 & Fabricación de muebles & C3120 & 16 \\
\hline 9 & Fabricación de otros productos mineros no metálicos & C2395 & 20 \\
\hline 10 & Fabricación de papel, productos de papel y cartón & C1709 & 7 \\
\hline 11 & Fabricación de productos de caucho y plástico & C2221 & 51 \\
\hline 12 & Fabricación de productos de metal excepto MyE & C2599 & 28 \\
\hline 13 & Fabricación de productos farmacéuticos & C2100 & 11 \\
\hline 14 & Fabricación de productos metalúrgicos básicos & C2431 & 14 \\
\hline 15 & Fabricación de productos textiles & C1311 & 46 \\
\hline 16 & Fabricación de sustancias y productos químicos & C2023 & 31 \\
\hline 17 & Fabricación de vehículos automotores & C2930 & 8 \\
\hline 18 & Instalación y mantenimiento de maquinaria & C3312 & 6 \\
\hline 19 & Otras industrias manufactureras & C3250 & 15 \\
\hline 20 & Transformación de la madera y fabricación & C1630 & 7 \\
\hline
\end{tabular}

Fuente: Cálculos propios

La investigación se desarrolla en dos etapas que exigen sean analizadas de manera consecuente. En la primera etapa se analizan los datos del SIREM, registrada en la Superintendencia de sociedades, en esta base de datos se encuentra la información financiera de las empresas formalmente constituidas para todo el territorio colombiano. Se realiza un análisis exploratorio de tipo visual para encontrar a priori las aglomeraciones que existan de acuerdo a cada actividad industrial. No obstante, en la etapa formal, se emplea un algoritmo para determinar si existe o no aglomeración, por medio de la prueba de Aleatoriedad Espacial Completa (CSR). A partir de la existencia de aglomeración se procede con la siguiente etapa, únicamente para los sectores industriales aglomerados.

Esta segunda etapa se concentra en el análisis de la productividad por sector industrial en el departamento de Antioquia. El proceso de determinación de la productividad en las actividades industriales en el departamento de Antioquia es aplicado para sectores que presenten un nivel de aglomeración tipo clúster. Para ello, se evalúan las variables Productividad, Capital y Trabajo.

\subsection{Determinación de aglomeración en Antioquia}

A partir de un proceso a priori, la determinación de aglomeración es posible visualizarla en el siguiente gráfico: un mapa coroplético que representa espacialmente la concentración del volumen de empresas en los municipios del departamento de Antioquia. 
Gráfico 3

Mapa coroplético de empresas por municipio

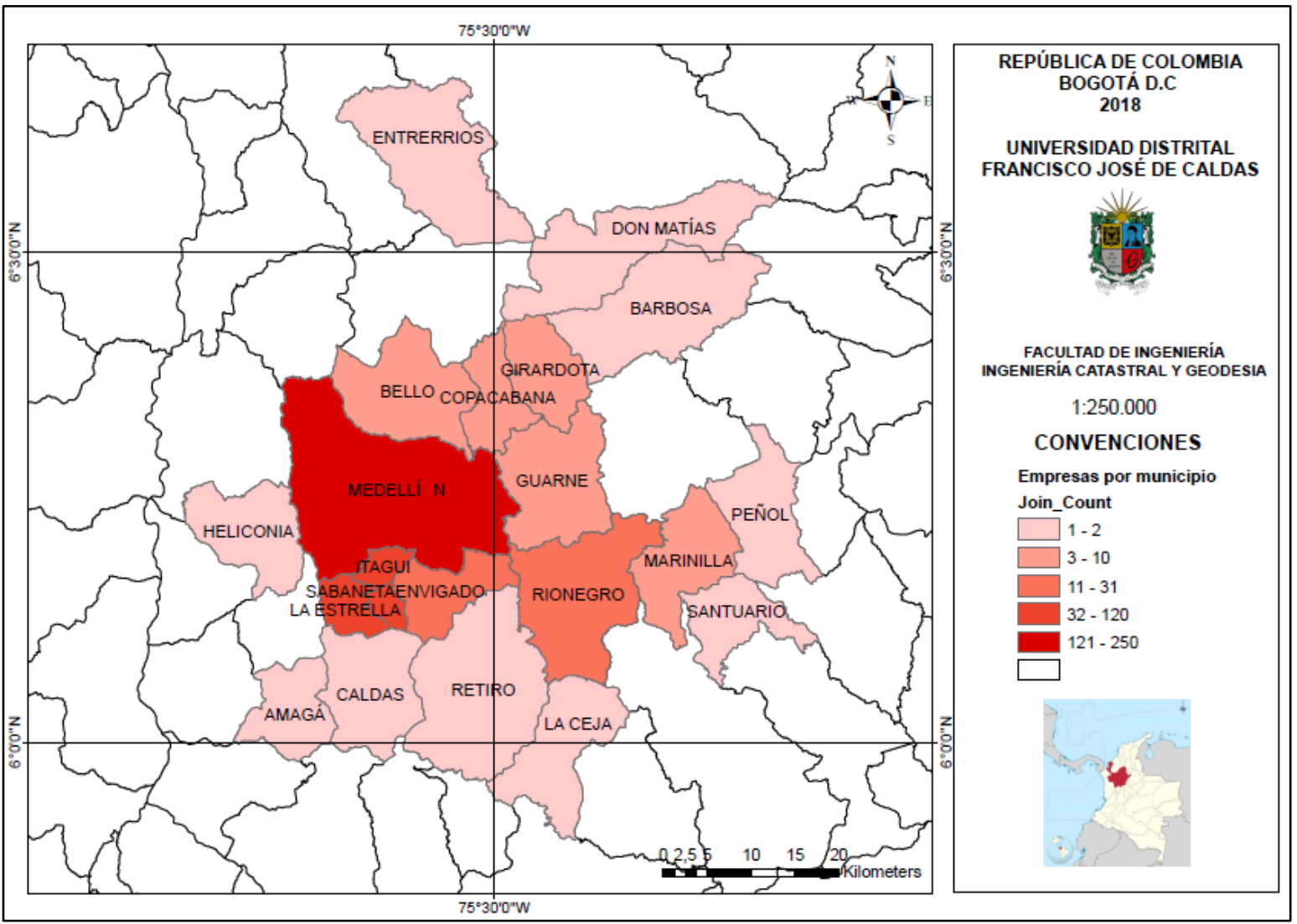

Fuente: cálculos propios

Como se puede apreciar en el mapa, la mayor concentración de la actividad industrial se registra en el Valle de Aburrá: comprendido por los municipios de Medellín Itagüí, La Estrella, Sabaneta, Envigado, Caldas y Bello. Cabe resaltar que existen otros municipios como Heliconia, Amagá, Rionegro, Guarne, Marinilla, Urrao, La Ceja, Yarumal, Entrerríos, Santuario y Don Matías; contiguos al área metropolitana del Valle de Aburrá, que también ejercen actividades industriales, no obstante, son significativamente menos intensivos.

Por otro lado, mediante el software ArcGIS es posible determinar aglomeración espacial a partir de la función de análisis de patrones puntuales llamada Average Nearest Neighbor (Vecino más cercano). Dicha herramienta mide la distancia entre cada centroide del punto y la ubicación del centroide de su vecino más cercano. Si la distancia promedio es menor que el promedio de una distribución hipotética aleatoria, se considera que la distribución de las entidades que se analiza es agrupada. En este sentido, los resultados son los esperados dado que el siguiente reporte nos comprueba que las empresas industriales en Antioquia se encuentran aglomeradas. Al analizar el $p$-value $<5 \%$, que rechaza la hipótesis nula, es decir, que los datos se encuentran distribuidos espacialmente de forma aleatoria. Al ser menor al 5\%, significa que la industria en Antioquia sigue un patrón de agrupamiento tipo clúster. El siguiente gráfico nos comprueba dicha hipótesis. 


\section{Gráfico 4}

Aglomeración industrial por método de Vecino más cercano

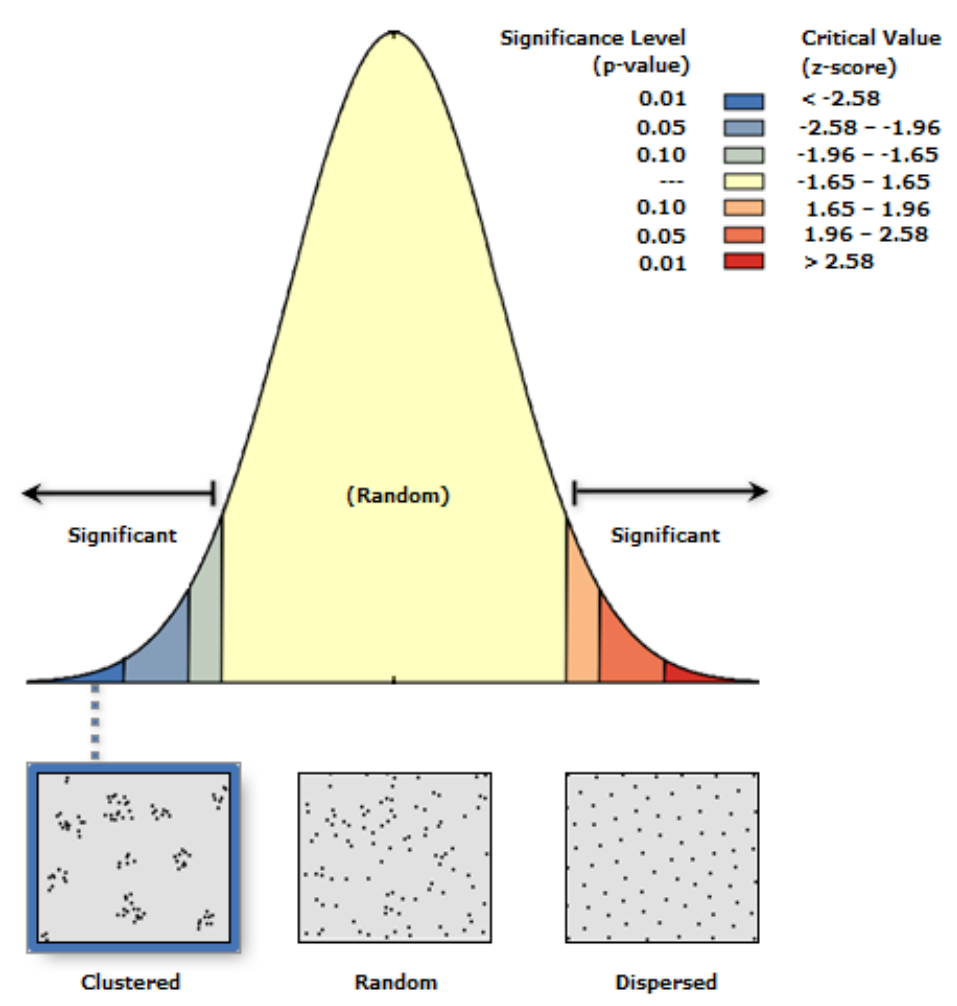

Fuente: Cálculos propios

Posteriormente se realiza la evaluación de la aglomeración espacial de las empresas industriales, empleando un método de estadística espacial basado en patrones puntuales por medio de una prueba gráfica denominada Aleatoriedad Espacial Completa (CSR). La característica de este es que se utiliza para datos muestreados como una variable discreta. La ubicación se realizó empresa por empresa, con el fin de saber si su distribución obedece a tres escenarios: 1) que los datos se encuentren agrupados formando clúster. 2) Los datos se encuentren localizados espacialmente de forma estocástica sin ningún patrón y, 3) Que los datos se encuentren ubicados de forma regular.

Para la determinación de aglomeración se emplea el análisis de la distribución de las localizaciones, se pretende averiguar si están distribuidas al azar en la región de estudio. Para este efecto se emplea la prueba visual de Aleatoriedad Espacial Completa (CSR). La prueba desarrollada en el software R-Studio por (Ortega \& Pérez, 2015) permite evaluar "si las localizaciones observadas estarán generadas al azar, es decir no siguiendo ningún patrón, siempre y cuando las diferencias entre su función de distribución empírica y este método teórico no sean significativas" 


\section{Gráfico 5}

Prueba visual de Aleatoriedad Espacial Completa (CSR)
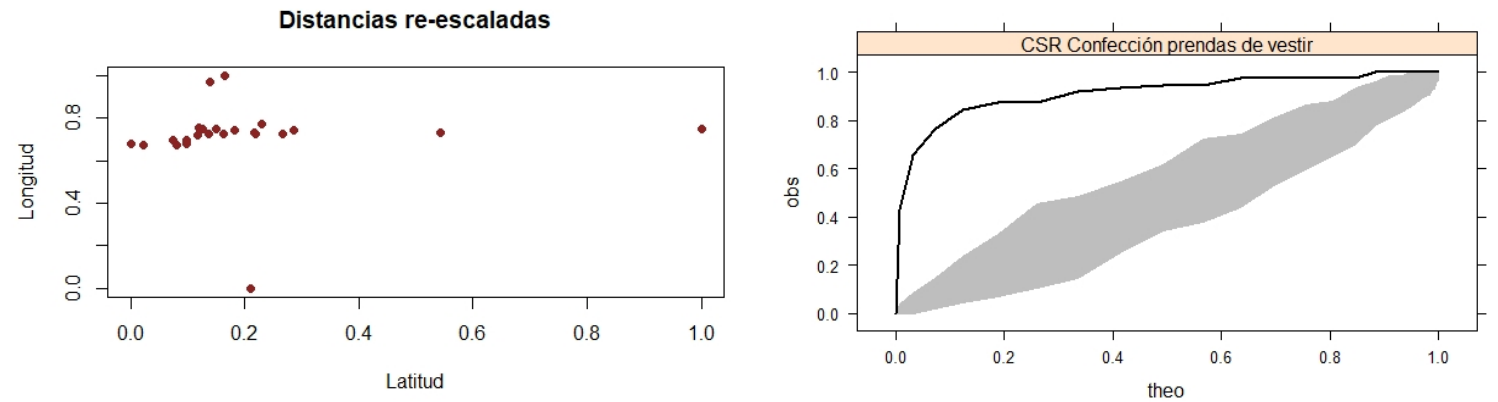

Fuente: Calculos propios a partir de Algoritmo de (Ortega \& Pérez, 2015)

Antes de obtener el resultado final, lo que se realizó primero fue una estandarización entre 0 y 1 de las coordenadas proyectadas. Dicha estandarización se observa en la gráfica de la izquierda, donde los puntos rojos representan las empresas que se dedican a las actividades de manufactura textil y que, se aglomeran sobre longitudes entre de 0.5 - 0.6 y sobre latitudes entre 0.0 - 0.3. Posteriormente, mediante la técnica CSR (Aleatoriedad Espacial Completa, por sus siglas en inglés) se adapta a cada sector industrial para determinar cuál de ellos se encuentra aglomerado, aleatorio o uniforme.

La gráfica de la derecha es la prueba concluyente para determinar aglomeración tipo clúster. Esta prueba es el resultado de la formalización matemática de un proceso de Poisson homogéneo de parámetro $\lambda$, el cual tiene en cuenta las distancias más cercanas entre puntos. El gráfico muestra tanto el modelo teórico que sigue una distribución espacial aleatoria (franja gris), mediante un proceso de simulación uniforme y, el modelo empírico (representado en la curva de color negro). Si la curva tiene el comportamiento como en la gráfica, indica que están aglomeradas las empresas y que se concentran alrededor de la latitud $0.0-0.1$ y la longitud $0.6-0.8$. Si esta curva se hubiese ubicado sobre la franja gris, es indicio de que no existe aglomeración tipo clúster, al contrario, sigue una distribución aleatoria completa.

\subsection{Modelo econométrico y estimación}

La estimación de la incidencia de la aglomeración industrial se realizó para 6 de las 20 actividades económicas que componen el sector industrial en el departamento de Antioquia. Se emplea la función de producción Cobb Douglas (Gujarati, 2010) que, en su forma estocástica, se expresa como:

$$
Y_{i}=\beta_{0} X_{1}^{\beta 1} X_{2}^{\beta 2} e^{u_{i}} \quad \text { Donde } \beta 1+\beta 2=1
$$

A pesar de que el modelo es completamente claro para ser empleado en la evaluación de la productividad, se debe realizar un ajuste a este modelo, de tal manera que sea expresado matemáticamente en forma lineal para poder estimar el modelo de Mínimos Cuadrados Ordinarios (MCO). Por tanto, al transformar el modelo se emplea la función logarítmica, obteniendo el resultado siguiente:

$$
\begin{aligned}
& \operatorname{Ln} Y_{i}=\ln \beta_{0+} \beta_{1} \ln X_{1 i}+\beta_{2} \ln X_{2 i}+u_{i} \\
& \operatorname{Ln} Y_{i}=\beta_{0+} \beta_{1} \ln X_{1 i}+\beta_{2} \ln X_{2 i}+u_{i}
\end{aligned}
$$

Donde las variables empleadas y sus significados son los siguientes: 
La anterior ecuación corresponde a la linealización de parámetros por logaritmos naturales de la función CobbDouglas, la cual permite el cálculo de elasticidades que representan los cambios relativos de la producción ante cambios relativos del capital y del trabajo.

En donde:

$Y=$ Producción: la proxy empleada aquí corresponde a los ingresos de actividades ordinarias: comprende los valores recibidos y/o causados como resultado de las actividades económicas desarrolladas bajo su razón social. Esta variable proviene del Estado de resultados integrales de acuerdo a las Normas Internacionales de Información Financiera (NIIF).

X1 = Factor capital: de acuerdo a la función neoclásica, la producción también depende del capital. Este factor de producción está relacionado con "las máquinas u otros elementos físicos que utilizan las empresas en el proceso de producción"(Sala-i-Martin, 2000). Para efectos del presente modelo se utilizará como proxy la cuenta de Propiedad, Planta y Equipo; registrado en el Estado de situación financiera de acuerdo a las Normas Internacionales de Información Financiera (NIIF).

X2 = Factor trabajo: Se emplea como proxy de salarios los valores provenientes de la cuenta de pasivos corrientes, suministrado por el Estado de situación financiera de acuerdo a las Normas Internacionales de Información Financiera (NIIF). Se utiliza únicamente la partida de pasivo corriente dado que las no corrientes aplican para períodos de más de un año, esto no es viable ya que la estimación del modelo de productividad es de corte transversal.

Se toma la cuenta descrita anteriormente dado que algunos pasivos corrientes, tales como las cuentas comerciales por pagar y otros pasivos acumulados (devengados), ya sea por costos de personal o por otros costos de operación, integran el capital de trabajo utilizado en el ciclo normal de operación de la entidad (International Accounting Standards Board, 2015).

Finalmente, $\mathrm{u}=$ término de perturbación estocástica o error del modelo

\section{Resultados}

Con base en el proceso de determinación de aglomeración por medio de la prueba visual de Aleatoriedad espacial completa (CSR); únicamente 5 actividades se encuentran aglomeradas, es decir siguen un patrón de localización que no deriva de una ubicación estocástica y tampoco organizados de forma regular.

De los 6 sectores que superaron la etapa de aglomeración, se les realizó la estimación de su productividad a partir de la función de producción de Cobb Douglas. Esta función luego de linealizarla para poder aplicar Mínimos Cuadrados Ordinarios (MCO), arrojó los siguientes resultados que en efecto se les validó los supuestos de Normalidad, Heterocedasticidad y Multicolinealidad. 
Gráfico 6

Sectores industriales aglomerados en el departamento

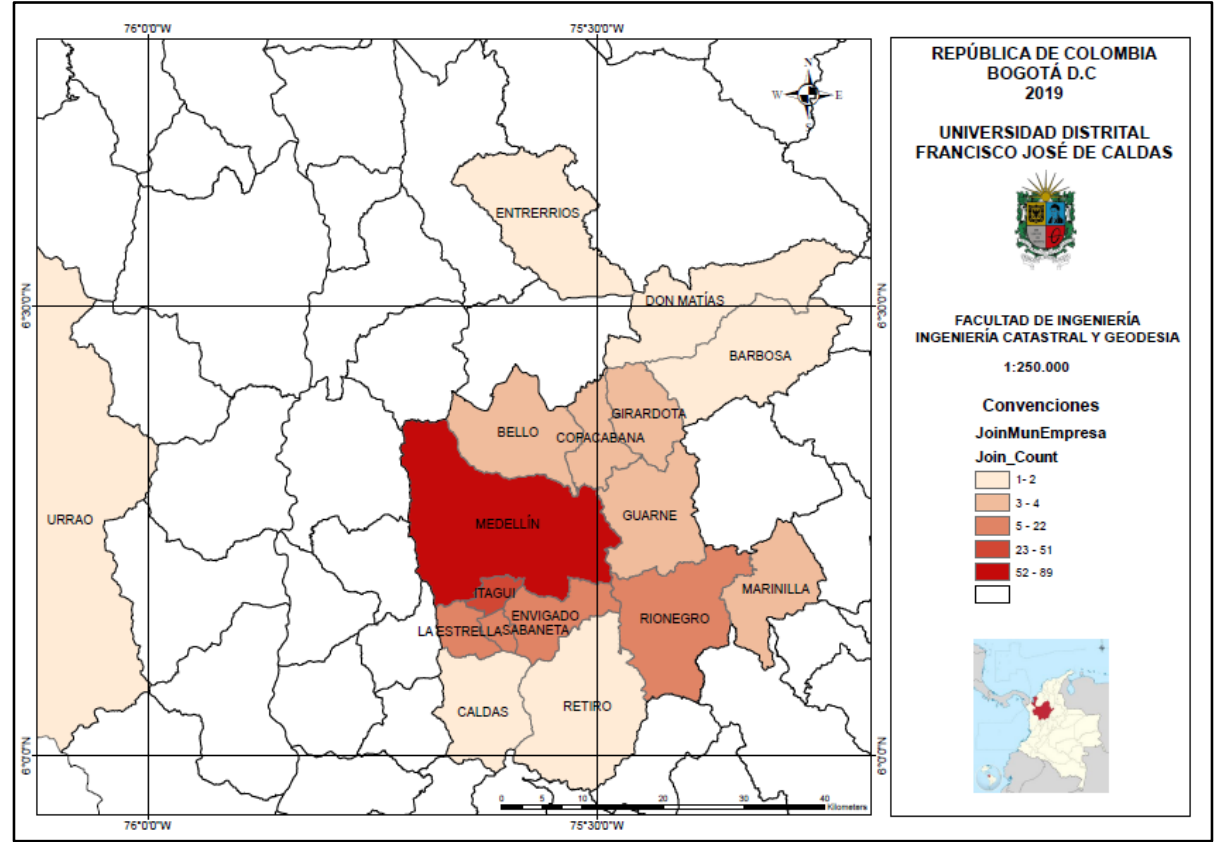

Fuente: Cálculos propios

Tabla 2

Estimación econométrica

\begin{tabular}{|r|l|l|l|l|r|l|}
\hline No & \multicolumn{1}{|c|}{ Sector } & Intercepto & \multicolumn{1}{|c|}{ Salario } & \multicolumn{1}{|c|}{ Capital } & $\begin{array}{c}\text { R2- } \\
\text { Ajustado }\end{array}$ & \multicolumn{1}{|c|}{$\begin{array}{c}\text { Significancia } \\
\text { global }\end{array}$} \\
\hline 1 & lonfección de prendas de vestir & $3.745^{* * *}$ & $0.745^{* * *}$ & $0.091^{* *}$ & 0.75 & $0.00^{* * *}$ \\
\hline 2 & Elaboración de productos alimenticios & $4.54^{* * *}$ & $0.625^{* * *}$ & $0.190^{*}$ & 0.76 & $0.00^{* * *}$ \\
\hline 3 & $\begin{array}{l}\text { Fabricación de productos elaborados con } \\
\text { metal excepto maquinaria }\end{array}$ & $4.13^{* *}$ & $0.5214^{* *}$ & $0.278^{* *}$ & 0.7 & $0.00^{* * *}$ \\
\hline 4 & Fabricación de productos textiles & $5.610^{* *}$ & $0.526^{* * *}$ & $0.184^{* *}$ & 0.5 & $0.00^{* * *}$ \\
\hline 5 & $\begin{array}{l}\text { Fabricación de sustancias y productos } \\
\text { químicos }\end{array}$ & $2.879^{*}$ & $0.679^{* * *}$ & $0.219^{* *}$ & 0.763 & $0.00^{* * *}$ \\
\hline 6 & Fabricación de productos de caucho & $1.697^{* *}$ & $0.838^{* * *}$ & $0.063^{* *}$ & 0.912 & $0.00^{* * *}$ \\
\hline
\end{tabular}

\footnotetext{
Nota: ${ }^{* *}$ Estadísticamente significativo al $1 \%$,

**Estadísticamente significativo al $5 \%$,

*Estadísticamente significativo al 10\%

Fuente: Cálculos propios
}

La tabla anterior muestra las estimaciones para los principales sectores industriales aglomerados, se tienen las estimaciones de parámetros para trabajo y capital, junto con sus respectivos p-value para determinar significancia individual y global, y el ajuste a partir de R cuadrado ajustado.

\subsection{Análisis de Resultados}

A partir de los resultados obtenidos en la determinación de presencia de aglomeración; se observa que, de los 20 sectores industriales, únicamente 6 de estos superaron la etapa de patrón de agrupamiento, es decir que al 
aplicarse la prueba de Aleatoriedad Espacial Completa (CSR), las empresas se concentran tipo clúster. Al analizar la gráfica 5, estos 6 sectores industriales se concentran en la región del Valle de Aburrá, siendo más intensos en la ciudad de Medellín y su área metropolitana que incluye Itagüí, Sabaneta, Envigado, La Estrella, Copacabana, Barbosa y Bello. No obstante, otros municipios contiguos también se ven beneficiados por la presencia de actividades afines.

La ciudad de Medellín es más intensiva en términos industriales que los demás municipios, ya que agrupa una gran parte de las actividades económicas industriales. La tradición de la actividad manufacturera en Antioquia, en especial las actividades relacionadas con la producción textil y confección de prendas de vestir, se le suma otras actividades que participan de esta cadena de valor como lo son la fabricación de productos de caucho, de productos de metal y fabricación de sustancias y productos químicos.

En cuanto a la estimación del modelo, es importante destacar que los estimadores $\left(\beta_{1} y \beta_{2}\right)$ corresponde con las elasticidades de las variables capital y trabajo, de tal forma que su interpretación sugiere una variación porcentual con respecto a la variable dependiente. Dichas estimaciones son significativas para los estimadores del trabajo y del capital. El ajuste de los modelos supera el 50\% para casi todos los modelos.

Además, la función de producción Cobb Douglas nos permite medir los rendimientos a escala: creciente cuando la suma de los estimadores de capital y trabajo son superiores a 1 ; constante cuando son iguales a $1 \mathrm{y}$ decrecientes cuando son inferiores a 1. De esta manera, se observa que, de las 6 actividades aglomeradas, 5 de ellas registran rendimientos decrecientes a escala; mientras que la actividad de Fabricación de productos de caucho, registra rendimientos constantes a escala.

De otro modo se observa que la industria en el departamento es intensiva en mano de obra, al ser industrias manufactureras, su mayor insumo es el trabajo. Mientras que la proporción de capital medido en términos de propiedad planta y equipo no genera un nivel de preponderancia sobre la actividad. Únicamente la actividad que registra mayor intensidad en Capital corresponde con el sector de Fabricación de productos de caucho. Con base en lo anterior, se observa la tradición manufacturera en Antioquia y en especial en la región metropolitana del Valle de Aburrá donde se desarrolla gran parte de la actividad industrial, con epicentro en la ciudad de Medellín.

\section{Conclusiones}

Antioquía sigue un patrón de aglomeración industrial, similar a la de los principales departamentos industriales en el país, es decir, es una industrialización en la ciudad capital con aglomeraciones y expansiones hacia sus centros poblados cercanos. La cercanía al principal centro de población, con acceso a mano de obra calificada y con mayores ventajas institucionales son elementos determinantes de la industria en el departamento de Antioquía, que permiten identificar ciertos patrones de aglomeración para textiles, confecciones, producción de alimentos, producción de metálicos, caucho, plástico y fabricación de sustancias y productos químicos. Este departamento ha mantenido una vocación industrial en Medellín y su área metropolitana y continúa siendo el eje productivo del departamento.

Se resalta la baja productividad marginal del capital y la alta productividad del trabajo en estas industrias, de las que se encuentran escalas de producción decrecientes o cercanas a un factor constantes a escala para la mayoría de ellas. Aunque la literatura de la aglomeración industrial argumenta que uno de los beneficios de dicha aglomeración es la posibilidad de acceder a economías de escala, al parecer y basado en el hecho de encontrar resultados que muestran rendimientos decrecientes o constantes, no hay evidencia de este tipo de economías para las industrias analizadas y aglomeradas en Antioquia.

Se hace necesario estudiar con mayor profundidad los nuevos patrones de localización, deslocalización, y relocalización de la industria en el departamento, a modo de identificar como los elementos institucionales tales 
como planes de ordenamiento, restricciones ambientales, e incentivos tributarios contribuyen a establecer nuevos patrones de aglomeración y muestran las nuevas dinámicas espaciales de la industria antioqueña.

\section{Referencias bibliográficas}

Artís, M., Ramos, R., \& Suriñach, J. (2007). Job losses, outsourcing and relocation: Empirical evidence using microdata. IZA Discussion Papers. http://www.econstor.eu/handle/10419/34731

Brouwer, A. E., Mariotti, I., \& Van Ommeren, J. N. (2004). The firm relocation decision: An empirical investigation. The Annals of Regional Science, 38(2), 335-347.

Botero Herrera, F. (1983). Antecedentes de la industrialización en Antioquia. Recuperado de http://tesis.udea.edu.co/handle/10495/4362

Camilo Murcia, J., Ricardo Amezquita, D., \& Javier Fuentes, H. (2014). Localización de la industria en Bogotá. UD y la GEOMÁTICA, 9.

Capello, R. (2014). Classical Contributions: Von Thünen, Weber, Christaller, Lösch. En M. M. Fischer \& P. Nijkamp (Eds.), Handbook of Regional Science (pp. 507-526). Springer Berlin Heidelberg. https://doi.org/10.1007/978-3-642-23430-9_94

Fuentes, H., Jimenez, L. C., \& Perez, N. (2019). La Demografía Industrial en Colombia: Localización y Relocalización de la Actividad Manufacturera. Cuadernos de Geografia, Vol. 28.

Fuentes, H. J, Amezquita, D. R., \& Murcia, J. C. (2014). Localización de la industria en Bogotá. UD y la geomática, (9), 75-86.

Gujarati, D. N. (2010). Econometría (5a. Ed.). McGraw-Hill Interamericana.

Holl, A. (2004). Transport Infrastructure, Agglomeration Economies, and Firm Birth: Empirical Evidence from Portugal*. Journal of Regional Science, 44(4), 693-712.

International Accounting Standards Board. (2015). Classification of Liabilities: Proposed amendments to IAS 1 : Comments to be received by 10 June 2015. IFRS Foundation.

Knoben, J., \& Oerlemans, L. A. G. (2008). Ties that spatially bind? A relational account of the causes of spatial firm mobility. Regional Studies, 42(3), 385-400.

Lampón, J. F. (2010). Deslocalización de la producción: Un análisis de los determinantes corporativos, logísticos y tecnológicos en el sector de fabricantes de componentes de automóvil. Universidad de Vigo.

Lee, Y. (2006). Relocation patterns in US manufacturing. FRB of Cleveland, Working Paper, 06-24.

Lee, Y. (2008). Geographic redistribution of US manufacturing and the role of state development policy. Journal of Urban Economics, 64(2), 436-450.

Londoño, C. (2015). Demografía industrial: Un estudio sobre relocalización de firmas en Colombia, 1995-2012. Lecturas de Economía, 82, 189-217.

Ocampo, J. A. (1984). Colombia y la economía mundial, 1830-1910. Siglo Veintiuno.

Ortega, M. Y. C., \& Pérez, A. G. (2015). Análisis estadístico de datos espaciales con QGIS y R (Edición: También en formato e-book). UNED. 
Ospina Vazquez, L. (1987). Industria y protección en Colombia: 1810 - 1930. FAES, Fondo Rotatorio de Publicaciones.

Pellenbarg, P. H., Van Wissen, L. Y. V. D., \& Van Dijk, J. (2002). J.(2002):“Firm migration”. Industrial.

Safford, F. (1965). Commerce and enterprise in central Colombia, 1821-1870. Columbia University.

Safford, F. (1989). El ideal de lo práctico: El desafío de formar una élite técnica y empresarial en Colombia. Empresa Editorial Universidad Nacional, El áncora editores.

Sala-i-Martin, X. (2000). Apuntes de crecimiento económico. Antoni Bosch editor.

Sleuwaegen, L., \& Pennings, E. (2006). International relocation of production: Where do firms go? Scottish Journal of Political Economy, 53(4), 430-446.

Supersociedades. (2018). Sistema de información empresarial.

Toro González, D. (2004). Localización de la industria manufacturera en Colombia 1990-1999.

Von Thünen, J. H. (1826). Isolated state.

Weber, A. (1929). Alfred Weber's theory of the location of industries. The University of Chicago Press.

Wood, A., \& Roberts, S. (2011). Economic Geography. Places, networks and flows (Fisrt). Routlegde.

Esta obra está bajo una Licencia Creative Commons

Attribución-NoCommercial 4.0 International

\section{(c) EY-NC}

